\title{
Low Frequency of Integrase Inhibitor Resistance Mutations Among Therapy-Naïve HIV Patients in Southeast China
}

This article was published in the following Dove Press journal:

Drug Design, Development and Therapy

\author{
Jinglan Lai \\ Yuming Liu \\ Xiao Han \\ Aiqiong Huang \\ Jin Lin \\ Wen Ao \\ Hanhui Ye \\ Yahong Chen
}

Department of Infectious Diseases, Mengchao Hepatobiliary Hospital of Fujian Medical University, Infectious Diseases Hospital of Fuzhou, Fuzhou, Fujian, People's Republic of China
Correspondence: Yahong Chen; Hanhui Ye Department of Infectious Diseases,

Mengchao Hepatobiliary Hospital of Fujian Medical University, No. 312, Xihong Road,

Fuzhou, Fujian, People's Republic of China $\mathrm{Tel}+8659188116119$

Fax +8659188116105

Email lucioleck@gmail.com; yehanhui@।63. com
Background: With the widespread use of integrase strand transfer inhibitors (INSTIs) in the clinical setting, transmission of INSTIs-resistance mutations may increase. Data regarding transmitted drug resistance mutations (TDRM) to INSTIs in Chinese HIV patients are limited. The aim of this study was to summarize the INSTIs TDRM, including the frequency of protease inhibitors (PIs) and reverse transcriptase (RT) inhibitors (RTIs) mutations in treatment-naïve patients in Southeast China.

Methods: HIV-1 positive patients were retrospectively selected between April 2018 and October 2020 from the Mengchao Hepatobiliary Hospital of Fujian Medical University, the largest designated HIV/AIDS care hospital in Southeast China. Individuals who were antiretroviral therapy-naïve and received antiretroviral drug resistance testing at baseline were included. Clinical data including demographic data, CD4 counts, HIV-RNA loads, and drug resistance mutations were collected.

Results: A total of 147 patients were enrolled. INSTIs TDRM was rare, with only one primary integrase mutation E138K observed in one sample and one secondary mutation E157Q detected in another sample. The overall prevalence of INSTIs TDRM was 1.36\%. A substantial proportion of patients harbored common INSTIs-associated polymorphic variants. Two samples harbored the T215S, M184V and K70E mutations related to nucleoside RTIs (NRTIs). Twelve patients carried nonnucleoside RTIs (NNRTIs)-resistance mutations. Two individuals harbored PIs-resistance mutations: Q58E in one patient and M46I, I54V, V82A, L10F, and Q58E mutations in another patient. The total TDRM rate for RTIs and PIs was $10.20 \%(15 / 147)$, but only $0.68 \%(1 / 147)$ was according to the WHO recommendations on TDRM.

Conclusion: The rate of INSTIs TDRM was low among therapy-naïve HIV patients in Southeast China. INSTIs as a first-line regimen are suitable for untreated HIV-1 patients in Southeast China. But special attention must be still paid to INSTIs TDRM in clinical practice. Keywords: HIV, transmitted drug resistance mutations, integrase strand transfer inhibitors, Southeast China

\section{Introduction}

Over the past decades, the extensive use of antiretroviral therapy (ART) for HIV patients has increased the incidence of TDRM. ${ }^{1}$ TDRM may result in treatment failure, disease progression, and mortality among newly infected HIV patients. Resistance against RTIs and PIs has frequently been determined in HIV patients. ${ }^{1-5}$

Attention to TDRM to INSTIs has gradually claimed increased interest after the widespread application of INSTIs. INSTIs are recommended as the first-line treatment regimens for HIV-1 patients, due to their high efficacy and good tolerability, 6,7 
and have been increasingly used in treatment-naïve patients with HIV since their introduction in China in 2009. In recent years, several studies on drug resistance to INSTIs have been declared in Mainland China and Taiwan. ${ }^{8-10}$ However, data on resistance to INSTIs in the ART-naïve population is insufficient in China. Furthermore, the prevalence of TDRM to INSTIs may vary across different regions due to different geographic and socio-economic conditions.

At present, no data related to INSTIs-resistance mutants have been reported in Southeast China. A better understanding of drug resistance against INSTIs is crucial for their efficient use in treatment regimens. Thus, our objective was to summarize INSTIs-resistance patterns including PIs and RTs mutations in treatment-naïve HIV patients in Southeast China.

\section{Materials and Methods Ethical Consideration}

The study was approved by the ethics committee of Mengchao Hepatobiliary Hospital of Fujian Medical University (The Ethics reference number: 2020-035-01). Existing clinical information and laboratory data were anonymously used and were abstracted from electronic medical records. Thus, the need for writing informed consent was waived.

\section{Study Population}

HIV-1 patients were retrospectively selected between April 2018 and October 2020 from those attending the Mengchao Hepatobiliary Hospital of Fujian Medical University, the largest designated HIV/AIDS care hospital in Southeast China. Individuals who were ART-naïve and had initial antiretroviral drug resistance screening test were included. Patients with HIV-RNA $<250 \mathrm{IU} / \mathrm{mL}$, HIV-2 infection, incomplete data, or previous exposure to ART were excluded.

\section{Data Collection}

Demographic information including sex, age, occupation, educational background, and transmission route was collected from medical records for each patient. Laboratory variables such as HIV-RNA loads, CD4 counts, and drugresistance data were further collected. All information was carefully checked after abstraction. CD4 counts were determined using the BD FACSCount system (Becton Dickenson, California, USA). Plasma HIV-RNA levels were quantitatively tested with the Ampliform HIV-1 Monitor Test, version 1.5 (Roche, Basel, Switzerland). The detection limit threshold was $<20 \mathrm{IU} / \mathrm{mL}$.

HIV drug-resistance test was carried out as follows: HIV-RNA was extracted from plasma samples using the QIAamp Viral RNA Mini Kit (Qiagen, Duesseldorf, Germany). The HIV gene was amplified using RT-PCR Kit (TaKaRa Biotechnology, Dalian, China).The obtained cDNA was amplified in a second round of nested PCR. After purification, the PCR products were sequenced by TSINGKE Biological Technology Co. Ltd (Beijing, China), and The sequences of amplified specific region were uploaded to GenBank.

Drug-resistant mutations were determined by interrogating the Stanford University HIV Drug Resistance Database (https://hivdb.stanford.edu/). The database defines DRM following four criteria including polymorphism frequency, treatment prevalence, in vitro phenotypes and association with VF. It provides an estimated level of resistance to a drug according to the penalty scores for the four criteria. There are five level of estimated levels: Susceptible, Potential low-level resistance, Low-level resistance, Intermediate resistance and High-level resistance.

\section{Results}

Among the 214 treatment-naïve patients, a total of 147 patients were evaluated in this study. Most were male patients $(95.92 \%)$ and the median age was 34 years (range, 18 to 83 years). The majority of patients acquired HIV by sexual transmission (48.98\% male/male, $31.29 \%$ male/female sexual contact). The median CD4 counts was 166 cells $/ \mu \mathrm{L}$ (range: $2-1004$ cells $/ \mu \mathrm{L}$ ). HIV-RNA loads ranged from $5.2 \times 10^{4} \mathrm{IU} / \mathrm{mL}$ to $3.7 \times 10^{7} \mathrm{IU} / \mathrm{mL}$. The most common subtypes were CRF01_AE and CRF07_BC (45.58\% and $42.18 \%$ ). Subtype B HIV-1 strains were determined in $4.76 \%$, subtype $\mathrm{C}$ in $2.72 \%$, and CRF08_BC in 2.04\%. CRF55_01B and CRF33_01B each accounted for $1.36 \%$ of the subjects (Table 1 ).

The rate of INSTIs TDRM was $1.36 \%$. Only one major INSTIs-resistance mutation (E138K) was detected in one sample. It suggested low-level resistance to raltegravir (RAL) and elvitegravir (EVG) and potential resistance to bictegravir (BIC) and dolutegravir (DTG). One accessory INSTIs-resistance mutation (E157Q) was observed in another sample, which can contribute to resistance if present along with other major resistance mutations resistance to RAL and EVG. The vast majority of samples harbored common INSTIs-associated polymorphic variants. The 
Table I Clinical Characteristics of Patients at Enrolment

\begin{tabular}{|c|c|}
\hline Variables & Patients (No, \%) \\
\hline \multicolumn{2}{|l|}{ Age (years) } \\
\hline $18-50$ & $110(74.83)$ \\
\hline $5 I-83$ & $37(25.17)$ \\
\hline \multicolumn{2}{|l|}{ Sex } \\
\hline Male & $14 \mid(95.92)$ \\
\hline Female & $6(4.08)$ \\
\hline \multicolumn{2}{|l|}{ Transmission route } \\
\hline MSM & 72 (48.98) \\
\hline Heterosexual & $46(31.29)$ \\
\hline Injection drug & I $(0.68)$ \\
\hline Unknown & $28(19.05)$ \\
\hline \multicolumn{2}{|l|}{ Marital status } \\
\hline Married & $78(53.06)$ \\
\hline Unmarried & $69(46.94)$ \\
\hline \multicolumn{2}{|l|}{ Ethnicity } \\
\hline Han & $147(100)$ \\
\hline \multicolumn{2}{|l|}{ CD4 count(cells/ $\mu \mathrm{L})$} \\
\hline$<200$ & $78(53.06)$ \\
\hline$\geq 200$ & $69(46.94)$ \\
\hline \multicolumn{2}{|l|}{ HIV RNA (IU/mL) } \\
\hline$<1 \times 10^{5}$ & $55(37.4 I)$ \\
\hline$\geq 1 \times 10^{5}$ & $92(62.59)$ \\
\hline \multicolumn{2}{|l|}{ Gene type } \\
\hline CRF_OIAE & $67(45.58)$ \\
\hline CRF_07BC & $62(42.18)$ \\
\hline B & $7(4.76)$ \\
\hline $\mathrm{C}$ & $4(2.72)$ \\
\hline CRF08_BC & $3(2.04)$ \\
\hline CRF55_0IB & $2(1.36)$ \\
\hline CRF33_0IB & $2(1.36)$ \\
\hline
\end{tabular}

Abbreviation: MSM, men who have sex with men.

most frequently observed polymorphic variants were T124A, T125A and S283G, followed by L101I, T112V, I135L, and K136Q.

In one participant, two NRTIs-resistance mutations (M184V, K70E) were observed. The M184V mutation can contribute to resistance if present along with other major resistance mutations high level resistance to lamivudine (3TC) and emtricitabine, and low-level resistance to didanosine and abacavir. NNRTIs-resistance mutations were most frequently identified. A total of 12 samples contained 13 NNRTIs-resistance mutations:V106I in 4 samples, V179E, V179D mutations each in 3 samples, E138A mutations in 2 samples and V106M in 1 sample. These resistance mutations can contribute to resistance if present along with other major resistance mutations potential low-level resistance to NNRTIs (Table 2).

Two patients harbored PIs-resistance mutations: Q58E mutation in one patient, 3 major mutations (M46I, I54V, V82A) and 2 accessory mutations (L10F, Q58E) in another individual (Table 2).

The total TDRM rate to RTIs and PIs was $10.20 \%$ $(15 / 147)$ with $0.68 \%(1 / 147)$ the World Health Organization (WHO) recommendation on TDRM. Only one patient had two-class drug resistance mutations. For the remaining 14 patients, one-class drug resistance mutations were detected.

\section{Discussion}

Studies regarding TDRM to INSTIs are not common, especially in China. We analyzed INSTIs-resistance patterns including mutations to RTIs and PIs widely used in HIV therapy-naive patients in Southeast China.

In our study, we identified the frequency of TDRM and polymorphisms in the HIV-1 integrase gene in 147 naïvetreatment patients from Southeast China. One major (E138K) and one accessory (E157Q) INSTIs-resistance mutation were detected in two different samples. A large proportion of ordinary polymorphic variants were detected. Most HIV-1 strains showed full susceptibility to the four most currently used INSTIs drugs. These findings were consistent with a previous study in China indicating that major INSTIs-selected TDRM are absent in INSTIstreatment naïve adult patients surveyed at the Tiantan Hospital. ${ }^{10}$ Several studies have also reported similar rates of INSTIs TDRM in treatment-naïve patients from other countries. In Italy, $0.2 \%$ of patients harbored INSTIs TDRM among 455 ART-naïve patients from 2006 to $2016 .{ }^{11}$ In South Korea, major INSTIs TDRM were identified in $3.4 \%$ of naïve patients. ${ }^{12}$ In Morocco, $5.2 \%$ of the study sample contained secondary mutations (L74IM, T97A), while no primary INSTIs-resistance mutations were detected among 77 ART-naïve patients. ${ }^{13}$ The prevalence of INSTIs-associated resistance mutations was $2.3 \%$ in Austrian patients recently diagnosed with HIV. ${ }^{14}$ In the United Kingdom, no major mutation for INSTIs was detected in treatment-naïve patients. ${ }^{15}$

We report for the first time the identification of the integrase E138K mutation associated with INSTIsresistance in HIV patients in China. This mutant confers low-level resistance to RAL and EVG, but sensitivity to DTG and BIC according to the Stanford University algorithm. Usually, E138K is a nonpolymorphic mutation 
Table 2 Distribution of Drug Resistance Mutations in Patients

\begin{tabular}{|c|c|c|c|c|c|c|}
\hline \multirow[t]{2}{*}{ Patients Code } & \multirow[t]{2}{*}{ Subtypes } & \multicolumn{4}{|c|}{ Mutation Type } & \multirow[t]{2}{*}{ Drug Resistance } \\
\hline & & INSTIs & NRTIs & NNRTIs & PIs & \\
\hline 8 & CRFOI_AE & & K70E, MI84V & & $\begin{array}{l}\text { M46I,I54V,V82A } \\
\text { LIOF, Q58E }\end{array}$ & FTC,3TC:H;DDI,D4T,TDF:L;ABC:I \\
\hline 13 & CRFOI_AE & & & V106I & & DOR:L; ETR,NVP,RPV:P \\
\hline 17 & CRF55-0IB & & & VI79E & & EFV,NVP,ETR,RPV:P \\
\hline 23 & CRFOI_AE & & & VI79D & & EFV,NVP:I; RPV:L; ETR:P \\
\hline 36 & CRF08_BC & & & EI38A & & ETR:P; RPV:L \\
\hline 37 & C & EI57Q & & & & EVG,RAL:P \\
\hline 38 & CRF07_BC & & & EI38A & & ETR:P; RPV:L \\
\hline 46 & CRFOI_AE & & & V106I & & DOR,ETR,NVP,RPV:P \\
\hline 50 & CRF07_BC & & & & Q58E & NFV/r:P; TPV/r:L \\
\hline 51 & CRFOI_AE & & & VI79D & & EFV,NVP,ETR,RPV:P \\
\hline 55 & CRF55_OIB & & & VI79E & & EFV,ETR,NVP,RPV:P \\
\hline 61 & CRFOI_AE & & & V106I & & DOR,ETR,NVP,RPV:P \\
\hline 69 & B & EI38K & & & & BIC,DTG:P; EVG,RAL:L \\
\hline 74 & CRFOI_AE & & & VI79D & & EFV,NVP,RPV,ETR: P \\
\hline 120 & B & & & VI06I & & DOR,ETR,NVP,RPV:P \\
\hline 145 & B & & & VI06M, VI79E & & DOR,ETR,NVP,RPV:P \\
\hline 147 & CRFOI_AE & & $\mathrm{T} 215 \mathrm{~S}$ & & & FTC,3TC:H;DDI,D4T,TDF:L;ABC:I \\
\hline
\end{tabular}

Abbreviations: INSTIs, intergrase strand transfer inhibitors; NRTIs, nucleoside reverse transcriptase inhibitors; NNRTIs, non-nucleoside reverse transcriptase inhibitors; Pls, protease inhibitors; H, high-level resistance; I, intermediate resistance; L, low-level resistance; P, potential resistance; FTC, emtricitabine; 3TC, lamivudine; DDI, didanosine; D4T, stavudine; TDF, tenofovir disoproxil fumarate; ABC, abacavir; DOR, doravirine; ETR, etravirine; NVP, nevirapine; RPV, rilpivirine; EVG, elvitegravir; RAL, raltegravir; NFV/r, nelfinavir/r; TPV/r, tipranavir/r; BIC, bictegravir; DTG, dolutegravir.

emerging in patients with virological treatment failure after receiving RAL, EVG, or DTG. ${ }^{16}$ Nevertheless, it also constitutes part of the natural genetic polymorphism of the IN gene. ${ }^{17}$ This mutation alone has not been reported to reduce susceptibility INSTIs treatment significantly, but may exhibit a great reduction in EVG and RAL susceptibility and intermediate-level resistance to BIC and DTG when it occurs in combination with other major INSTIs DRM. ${ }^{18}$

Another INSTIs-resistance mutation observed in our study was E157Q, which confers potential resistance to RAL and EVG, but sensitivity to DTG and BIC. Similar to the study conducted by Liu et $\mathrm{al}^{10}$, who first reported the presence of the E157Q mutation among Chinese ART-naïve patients in 2019, the frequency of the integrase E157Q mutation was very low in China. E157Q is a common polymorphic mutation selected by RAL treatment in HIV patients ${ }^{19}$ and by exposure to EVG in vitro. The prevalence of the E157Q polymorphic mutation was $2.7 \%$ in INSTIs-naïve patients from 17 French clinical centers, ${ }^{20}$ and $2.4 \%$ in ART-naïve patients in Italy. ${ }^{21}$ These studies have demonstrated that the E157Q mutation itself has minimal effects on RAL and DTG activity and viral infectivity. E157Q has been shown to act as a compensatory mutation due to its ability to restore damaged enzymatic activity caused by $\mathrm{N} 155 \mathrm{H}$ or R263K substitution. ${ }^{20-22}$

As displayed in our result, the frequency of TDRM to RTIs and PIs was also low. Two individuals were detected with resistance mutation to NRTIs. And PIs-resistance mutations were identified in two patients. However, 12 patients $(8.16 \%)$ harbored resistance to NNRTIs. The total TDRM rate to RTIs and PIs was $10.20 \%(15 / 147)$, including $0.68 \%$ (1/147) WHO recommendation DRM. These results were in accordance with previous reports indicating that TDRM to RTIs and PIs are not common in treatment-naïve patients living in China. ${ }^{23,24}$

In summary our study showed that the overall TDRM rate was low, and the frequency of INSTIs-resistance mutations was absent in our patient sample. However, the frequency of INSTIs-resistance mutations may increase with the extensive use of ART, since E138K, a major INSTIs-resistance mutation, was also detected in our study cohort. Thus, it is necessary to pay attention to the presence of TDRM, including those suggestive of INSTIs-resistance during baseline evaluations of patients during the routine clinical work-up.

Our study had some limitations. The small sample size may have resulted in a selection bias. Furthermore, the 
retrospective nature of the study limited the interpretation of the rate to TDRM. A large prospective study should be conducted to explore the frequency of TDRM in therapynaïve patients in Southeast China.

\section{Conclusion}

Our study demonstrated that the frequency of TDRM was low in our HIV patient cohort. However, TDRM associated with INSTIs-treatment resistance, including E138K and E517Q were also detected in therapy- naïve HIV patients in Southeast China. Thus, TDRM test should be conducted in untreated HIV patients in Southeast China before initiating ART.

\section{Funding}

This study was funded by grants from the Scientific Foundation of Fuzhou (grant no. 2019-SZ-43), the Key Clinical Specialty Discipline Construction Program of Fujian, P.R.C. and the Clinical Medicine Center Construction Program of Fuzhou, Fujian, P.R.C. (2018080306).

\section{Disclosure}

The authors declare that they have no competing interests.

\section{References}

1. Clutter DS, Jordan MR, Bertagnolio S, Shafer RW. HIV-1 drug resistance and resistance testing. Infect Genet Evol. 2016;46:292-307. doi:10.1016/j.meegid.2016.08.031

2. Wu KS, Tseng YT, Sy CL, et al. Prevalence of HIV-1 transmitted drug resistance and viral suppression among recently diagnosed adults in Sao Paulo, Brazil. BMC Infect Dis. 2019;164:699-706.

3. Weng YW, Chen IT, Tsai HC. Trend of HIV transmitted drug resistance before and after implementation of HAART regimen restriction in the treatment of HIV-1 infected patients in southern Taiwan. BMC Infect Dis. 2019;19:741.

4. Lunar MM, Zidovec Lepej S, Tomazic J, et al. HIV-1 transmitted drug resistance in Slovenia and its impact on predicted treatment effectiveness: 2011-2016 update. PLoS One. 2018;13:e0196670. doi:10.1371/ journal.pone. 0196670

5. Hofstra LM, Sauvageot N, Albert J, et al. Transmission of HIV drug resistance and the predicted effect on current first-line regimens in Europe. Clin Infect Dis. 2016;62:655-663. doi:10.1093/cid/civ963

6. Lehmann C, Malin J, Suarez I, Fatkenheuer G. [Modern HIV treatment]. Internist (Berl). 2019;60:411-419. doi:10.1007/s00108-019-0564-0. German.

7. Messiaen P, Wensing AM, Fun A, Nijhuis M, Brusselaers N, Vandekerckhove L. Clinical use of HIV integrase inhibitors: a systematic review and meta-analysis. PLoS One. 2013;8:e52562. doi:10.1371/journal.pone.0052562
8. Chang SY, Lin PH, Cheng CL, et al. Prevalence of Integrase Strand Transfer Inhibitors (INSTI) resistance mutations in Taiwan. Sci Rep. 2016;6:35779. doi:10.1038/srep35779

9. Lai CC, Liu WC, Fang CT, et al. Transmitted drug resistance of HIV-1 strains among individuals attending voluntary counselling and testing in Taiwan. J Antimicrob Chemother. 2016;71:226-234. doi:10.1093/jac/dkv284

10. Liu L, Dai L, Yao J, et al. Lack of HIV-1 integrase inhibitor resistance among 392 antiretroviral-naïve individuals in a tertiary care hospital in Beijing, China. AIDS. 2019;33:1945-1947. doi:10.1097/ QAD.0000000000002282

11. Rossetti B, Di Giambenedetto S, Torti C, et al. Evolution of transmitted HIV-1 drug resistance and viral subtypes circulation in Italy from 2006 to 2016. HIV Med. 2018;19:619-628. doi:10.1111/hiv.12640

12. Jeong W, Jung IY, Choi H, et al. Integrase strand transfer inhibitor resistance mutations in antiretroviral therapy-naive and treatment-experienced HIV patients in South Korea. AIDS Res Hum Retroviruses. 2019;35:213-216. doi:10.1089/aid.2018.0213

13. Alaoui N. Prevalence of resistance to integrase strand-transfer inhibitors (INSTIs) among untreated HIV-1 infected patients in Morocco. J Korean Med Sci. 2018;11:369.

14. Zoufaly A, Kraft C, Schmidbauer C, Puchhammer-Stoeckl E. Prevalence of integrase inhibitor resistance mutations in Austrian patients recently diagnosed with HIV from 2008 to 2013. Infection. 2017;45:165-170. doi:10.1007/s15010-016-0936-5

15. Tostevin A, White E, Dunn D, et al. Recent trends and patterns in HIV-1 transmitted drug resistance in the United Kingdom. HIV Med. 2017;18:204-213. doi:10.1111/hiv.12414

16. De Francesco MA, Izzo I, Properzi M, et al. Prevalence of integrase strand transfer inhibitors resistance mutations in integrase strand transfer inhibitors-naive and -experienced HIV-1 infected patients: a single center experience. AIDS Res Hum Retroviruses. 2018;34:570-574. doi:10.1089/aid.2018.0006

17. Lataillade M, Chiarella J, Kozal MJ. Natural polymorphism of the HIV-1 integrase gene and mutations associated with integrase inhibitor resistance. Antivir Ther. 2007;12:563-570.

18. Memarnejadian A, Nikpoor AR, Davoodian N, Kargar A, Mirzadeh Y, Gouklani H. HIV-1 drug resistance mutations among antiretroviral drug-experienced patients in the south of Iran. Intervirology. 2019;62:72-79. doi:10.1159/000501255

19. Malet I, Delelis O, Valantin MA, et al. Mutations associated with failure of raltegravir treatment affect integrase sensitivity to the inhibitor in vitro. Antimicrob Agents Chemother. 2008;52:1351-1358. doi:10.1128/AAC.01228-07

20. Charpentier C, Malet I, Andre-Garnier E, et al. Phenotypic analysis of HIV-1 E157Q integrase polymorphism and impact on virological outcome in patients initiating an integrase inhibitor-based regimen. $J$ Antimicrob Chemother. 2018;73:1039-1044. doi:10.1093/jac/dkx511

21. Saladini F, Giannini A, Boccuto A, Tiezzi D, Vicenti I, Zazzi M. The HIV-1 integrase E157Q polymorphism per se does not alter susceptibility to raltegravir and dolutegravir in vitro. AIDS. 2017;31:2307-2309. doi:10.1097/QAD.0000000000001616

22. Anstett K, Cutillas V, Fusco R, Mesplède T, Wainberg MA. Polymorphic substitution E157Q in HIV-1 integrase increases R263K-mediated dolutegravir resistance and decreases DNA binding activity. J Antimicrob Chemother. 2016;71:2083-2088. doi:10.1093/jac/dkw109

23. Zhao S, Feng Y, Hu J, et al. Prevalence of Transmitted HIV drug resistance in antiretroviral treatment naïve newly diagnosed individuals in China. Sci Rep. 2018;8:12273. doi:10.1038/s41598-018-29202-2

24. Hao M, Wang J, Xin R, et al. Low rates of transmitted drug resistances among treatment-naive HIV-1-infected students in Beijing, China. AIDS Res Hum Retroviruses. 2017;33:970-976. doi:10.1089/ aid.2017.0053 


\section{Publish your work in this journal}

Drug Design, Development and Therapy is an international, peerreviewed open-access journal that spans the spectrum of drug design and development through to clinical applications. Clinical outcomes, patient safety, and programs for the development and effective, safe, and sustained use of medicines are a feature of the journal, which has also been accepted for indexing on PubMed Central. The manuscript management system is completely online and includes a very quick and fair peer-review system, which is all easy to use. Visit http://www. dovepress.com/testimonials.php to read real quotes from published authors. 